\title{
Monachisme et reconfigurations du style chrétien : à propos du Temps des moines
}

Alain Rauwel

\section{(2) OpenEdition \\ 1 Journals}

\section{Édition électronique}

URL : https://journals.openedition.org/assr/29741

DOI : 10.4000/assr.29741

ISSN : $1777-5825$

Éditeur

Éditions de l'EHESS

\section{Édition imprimée}

Date de publication : 1 décembre 2017

Pagination : 243-248

ISSN : 0335-5985

\section{Référence électronique}

Alain Rauwel, «Monachisme et reconfigurations du style chrétien : à propos du Temps des moines », Archives de sciences sociales des religions [En ligne], 180 | octobre-décembre 2017, mis en ligne le 01 décembre 2017, consulté le 22 septembre 2021. URL : http://journals.openedition.org/assr/29741 ; DOI : https://doi.org/10.4000/assr.29741 


\section{Alain Rauwel}

\section{Monachisme et reconfigurations du style chrétien}

Il peut sembler paradoxal d'aborder la question du christianisme en modernité avancée, qui est de longue date l'interrogation majeure de Danièle Hervieu-Léger, en se donnant pour objet d'examen, au fil d'un gros livre, le monachisme: comment en effet prendre la mesure de ce que signifie l'engagement religieux dans une société d'exculturation chrétienne au prisme d'une forme de vie qui s'est très longtemps définie comme fuga mundi, refus radical du "monde » en ce qu'il est socialisation non ecclésiale? En outre, comment poser la question de la modernité dans un univers canoniquement clos qui a tendance à se construire comme soustrait à l'historicité du siècle, installé par son ancrage liturgique et sa visée eschatologique aux confins du muable et de l'éternel ? Le choix de s'inscrire dans cette temporalité singulière qu'est «le temps des moines » est-il donc sociologiquement pertinent ?

À cette mise en demeure méthodologique, D. Hervieu-Léger répond... par l'histoire! Une grande part de son effort consiste à extraire le monachisme de sa gangue de fixité supposée ( "stat crux dum volvitur orbis» est, comme on sait, la devise des Chartreux), y compris et surtout quand des moines eux-mêmes en font un argument apologétique. Elle est d'autant plus fondée à le faire que la chronologie retenue pour son étude en manifeste à deux niveaux la nécessité: premièrement, parce que, dans la France de 2017, il n'est de vie monastique masculine que "restaurée ", c'est-à-dire entièrement réinventée, au XIX siècle; aucun établissement n'a franchi indemne la césure révolutionnaire, autorisant à fantasmer une continuité séculaire ou millénaire; deuxièmement, parce qu'à l'échelle même d'une vie de moine, le cénobitisme de notre temps n'est plus, loin s'en faut, celui des années 1950. En même temps que les modalités du quotidien évoluaient en profondeur, l'enthousiasme qui faisait encore, il y a quelques décennies, élever des bâtiments propres à accueillir de vastes communautés (Landévennec), s'est teinté d'inquiétude. Les plus pessimistes reprendraient volontiers les mots de Renan au Mont-Cassin en 1849: "Grands corridors, réfectoires vides. Pauvre vie monastique! C'en est fait d'elle ${ }^{1}$.» Et les optimistes ne tablent pas sur une persistance impossible, mais sur une inévitable mutation. Au long des deux derniers siècles, en tout cas, la vie régulière n'a cessé de connaître des reconfigurations, qui en font un laboratoire d'invention sociale permanente. Tel est en somme l'objet central du livre de D. Hervieu-Léger.

Ce fait explique qu'un grand livre de sociologie soit rempli d'analyses historiques. Le défi n'était pourtant pas mince, tant l'historiographie du monachisme

1. E. Renan, Voyage en Italie, Paris, Arléa, 1999, p. 53. 
contemporain souffre d'un handicap sévère: elle est très majoritairement autoproduite par des auteurs issus de la sphère cénobitique, qui tendent à diffuser une « histoire sainte », avec d'autant plus d'autorité que les règles de l'histoire savante moderne sont de fabrication monastique (Mabillon) et que les successeurs des Mauristes, de quelque robe qu'ils soient, maîtrisent généralement les codes rhétoriques qui font conclure à de la «bonne histoire». Le sentiment de propriété des moines sur leur histoire est saisissant; dans des groupes fermés où tout se légitime par l'auctoritas Patrum, l'enjeu est certes vital. La manière dont les Cisterciens ont, au gré de récentes commémorations (1990, 1998,2003), tenté de réaffirmer leur leadership sur les études bernardines, avec la collaboration volontaire ou involontaire de chercheurs étonnamment respectueux, en dit long sur la difficulté de travailler sereinement sur ces sujets. Il n'est d'ailleurs pas nécessaire d'avoir neuf siècles d'âge pour pratiquer et imposer la langue de buis historique: la spécialité belge qu'est l'apologie à jets continus de Lambert Beauduin, le fondateur de Chevetogne, ou l'opacité totale qui règne sur la véritable histoire de Taizé en sont parmi d'autres des preuves éclatantes, sans parler de l'hagiographie pure telle qu'elle est mobilisée par les moines du Barroux, qui ont très logiquement doublé leur abbatiale néo-romane d'un récit de fondation tout droit sorti du $\mathrm{XI}^{\mathrm{e}}$ ou du $\mathrm{XII}^{\mathrm{e}}$ siècle. Le mérite de D. Hervieu-Léger est donc certain, d'avoir tracé son sillon entre des murailles d'études promotionnelles, qu'il s'agisse de Prosper Guéranger à Solesmes, de Jean-Baptiste Muard à La Pierre-qui-vire, ou de cet étrange apôtre de la Champagne pouilleuse que fut Ernest André. Un trait commun à tous ces cénobitismes dix-neuviémisants est l'absence de formation proprement monastique des fondateurs, imposant déficit de continuité et de tradition et édification sur des bases autres: alors qu'aucun parmi les anciens Mauristes ne réussit à reprendre du service, Guéranger venait du clergé diocésain, Muard aussi, animé en outre par une visée missionnaire peu compatible avec le primat de la contemplation, et on doit à André la conjonction des deux identités closes de l'abbaye et du village en un «monachisme paroissial» pour le moins inédit.

Le fondateur du Mesnil-Saint-Loup entendait ramener les fidèles à la maison du Père d'abord par la pratique assidue de la liturgie. Le culte public n'est pas seulement en effet l'une des notes du monachisme mais son centre vital, autour duquel tout le reste gravite. C'est l'une des grandes forces du beau livre de D. Hervieu-Léger que d'affronter sans échappatoires cette donnée qui effraie généralement les sociologues. Il faut le reconnaître: alors même que l'anthropologie a produit de nombreuses théories du rite, les sciences sociales peinent, lorsqu'elles sont confrontées sur les terrains géographiquement proches à des phénomènes rituels, à les inscrire à leur vraie place. Le vaudou ou le culte des ancêtres semblent épistémologiquement moins irritants que la messe ou l'office divin. Que faire alors sinon, comme D. Hervieu-Léger, se donner ce mot d'ordre très simple: "observer la liturgie». Bien des échecs viennent d'une volonté de typologiser trop vite. En matière cultuelle, la gleichschwebende Aufmerksamkeit de l'analyste s'impose. Les lieux de la liturgie fournissent une excellente entrée; leur disposition matérielle en dit long sur la manière dont la communauté étudiée comprend son activité principale, sa raison d'être même. Le Temps des moines offre des pages remarquables sur les procédures de séparation ou de rassemblement des moines et des fidèles de passage dans l'église ou l'oratoire. Toutes les 
configurations sont ici envisageables, du partage des mêmes bancs à l'existence d'une gigantesque grille barrant l'abbatiale et y réservant, tout au fond, une sorte de «cage » pour des visiteurs à peine admis à contempler de loin la performance des virtuoses - éloignement qui peut aussi bien être sonore, selon la formulation délicieusement révélatrice d'une pancarte jadis placardée à Solesmes: "On est prié de ne pas unir sa voix au chour des moines ${ }^{2}$." Tout observateur, même très éphémère, perçoit nettement quelle conception de l'Église prévaut dans l'édifice ainsi structuré: nous sommes bien, comme le dit D. Hervieu-Léger, dans le domaine de l'«ecclésiologie pratiquée».

C'est pourquoi la «question liturgique» n'est pas une affaire de techniciens, un détail sur lequel on pourrait passer vite. Dans les monastères, elle suscite, et surtout a suscité, entre 1965 et 1975 notamment, des débats d'autant plus violents qu'ils traversaient un monde silencieux par vœu. Les réaménagements des chœurs, le passage du latin au vernaculaire, l'introduction de textes et de gestes nouveaux: tout cela a fait trembler sur leurs bases bien des vocations cénobitiques, précisément parce que dans le rituel c'est le cœur même du propositum qui était remis en cause. La hiérarchie interne s'en trouvait aussi bouleversée, par l'abolition de la distinction entre moines de chœur et convers et par la remise en cause, conforme à la Règle, de l'ordination sacerdotale systématique. D. Hervieu-Léger, qui a l'art consommé d'entendre entre les phrases, restitue à juste titre «des communautés au bord de la rupture». Tant de choses se disent d'un groupe par son art de célébrer. Point n'est besoin même de voir: la multiplication des enregistrements révèle à l'oreille attentive des théologies autant que des anthropologies différenciées. Quelle distance d'un chœur solesmien jouant sur l'unisson parfait, les voix blanches et dégenrées, à une communauté autrefois prospère mais réduite en nombre et en forces qui accepte de faire entendre les brisures de voix vieillies ou fatiguées! "Monachisme ostensible ", comme dirait D. Hervieu-Léger, face à un «monachisme kénotique », comme on eût dit il y a quarante ans.

La question de la temporalité, qui est un fil rouge de l'étude, trouve dans la liturgie l'un de ses meilleurs tests. Le propre de la ritualité est en effet, tout en insérant du hors-temps dans les rythmes communs, de jouer sur le principe de répétition. Le moine en est le témoin le plus assidu: chaque jour à Laudes revient le Benedictus, chaque jour à Vêpres revient le Magnificat, le cycle du psautier ne demande que peu de jours ou de semaines pour être parcouru dans son entier. Il faut que le cœur même du kérygme soit en cause pour que, pendant le triduum pascal, l'ordre des heures soit brièvement modifié. On comprend par ce biais pourquoi des tentatives comme celle de Boquen, désormais bien connue ${ }^{3}$ et à laquelle D. Hervieu-Léger consacre des pages exceptionnellement informées, sont sans doute sorties plus précocement que ne le pensaient les protagonistes eux-mêmes de l'orbe monastique. "Chaque cérémonie était un événement", dit Bernard Besret du Boquen qu'il a porté à l'avant-scène du néo-catholicisme des seventies: y a-t-il plus contradictoire avec l'éternel retour des mots et des gestes qui tisse le

2. Cité par L. Bouyer, «Réflexions sur le mouvement liturgique », Dieu vivant, 19, 1951, p. 84. 3. B. Lebel-Goascoz, Boquen: entre utopie et révolution dans le catholicisme français, Rennes, Presses universitaires de Rennes, 2015. 
temps communautaire en terrain bénédictin? Boquen fut, de ce point de vue, un «lieu d'Église ${ }^{4}$ » beaucoup plus qu'une maison cistercienne, même chamboulée.

L'antithèse de la nouveauté permanente, on la trouvera bien sûr dans les monastères de la sphère "tradi », sur lesquels D. Hervieu-Léger donne un chapitre particulièrement attendu. Le phénomène est en effet d'autant plus constamment évoqué (ou invoqué) qu'il est rarement étudié: voici enfin des propositions sérieuses pour comprendre et remettre à leur vraie place des fondations dont les tenants du monachisme ancien ne sont pas les derniers à parler, se demandant ce qu'ils ont pu faire au bon Dieu pour que des groupuscules nés de la dernière pluie, mais cultivant avec des soins d'antiquaires les coutumes d'avant-hier, rencontrent des succès en apparence spectaculaires. Si des jeunes gens issus de milieux très homogènes choisissent Fontgombault ou Le Barroux de préférence à telle petite Trappe rustique, rappelle l'auteur, c'est un choix au moins aussi hyper-moderne qu'anti-moderne: qu'est-ce en effet que préférer telle façon de vivre sui generis à l'objectivité de l'ascèse en Église sinon sacrifier au dogme principal de la modernité avancée, la primauté du subjectif? On observera d'ailleurs que le dandysme qui rentre beaucoup plus qu'on ne croit dans l'alchimie de nombreuses "vocations " régulières peut tout aussi bien jouer à l'envers: par maximalisme, un jeune bourgeois très diplômé entrera dans une communauté de moines-fermiers... et la quittera généralement assez vite. Il en va de même pour le recours aux ressources mondaines: rien n'empêche de se donner un cadre de vie intégralement néo-médiéval et d'en faire la promotion en jouant sur tous les ressorts de la civilisation geek. L'urbain connecté de 2017 peut charger sur son smartphone une application qui lui fera entendre, en direct ou en différé, le grégorien «éternel » psalmodié sur une colline de Provence et sous des voutes «romanes » achevées en 1989 ! Échappe-t-on aux contradictions de son siècle en mettant en scène la fuite $d u$ siècle? Le Temps des moines montre que les choses ne sont pas si simples.

Les failles ne séparent pas seulement un monachisme anté-conciliaire d'un monachisme conciliaire, pour le dire trop schématiquement. Elles passent aussi et surtout entre maisons et familles religieuses, alors même que des identités communes sont hautement revendiquées. D. Hervieu-Léger, pour avoir sillonné les chemins des monastères français, rend avec une maestria impressionnante la diversité des situations locales. Mieux, elle construit en objet sociologique une réalité qui échappe de toutes parts à la généralisation. Comme l'a rappelé Giorgio Agamben, scientia monastica signifie originairement «science des vies singulières ». Si le cadre institutionnel de chaque monastère est serré, celui des congrégations est assez lâche pour permettre tous les cas d'espèce. Chez les bénédictins «noirs ", l'abbé-primat, de création assez récente, exerce un ministère d'unité plus qu'un réel gouvernement. Les congrégations, de Solesmes d'un côté, de Subiaco de l'autre, ont plus de réalité quotidienne, ne serait-ce que par le jeu des visites canoniques. Mais leur unité interne ne résiste guère à l'examen. Solesmes n'est pas Fontgombault et, pour des raisons diamétralement opposées, n'est pas non plus Ligugé, qui lui-même n'est pas Saint-Wandrille. Tous ces établissements sont pourtant supposés être dans un rapport de filiation. Il y a sans doute davantage de cohésion à l'intérieur de la congrégation de Subiaco, mais aussi entre Subiaco et

4. Ph. Boitel, Lieux d'Église, Paris, Éditions du Seuil, 1975. 
les Cisterciens, au point que, les couleurs d'habits se faisant discrètes, il est parfois difficile de distinguer "à l'aveugle» une Trappe et une maison de moines noirs. Restent des cas irréductibles à tout classement, parmi lesquels D. Hevieu-Léger distingue celui de Sept-Fons. On lira avec la plus grande attention le dossier riche et neuf qu'elle réunit sur cette communauté tendant jusqu'aux limites de la rupture les potentialités d'emprise de la vie régulière (invention locale d'une sainteté dogmatique, forte autorité abbatiale, réduction massive de l'espace privé, etc.); la possibilité de pérenniser un tel modèle dans un monde catholique de plus en plus soucieux, au moins dans le discours, de la protection des droits individuels, sera un intéressant objet d'observation dans les années à venir.

Forte de sa riche moisson d'observations, D. Hervieu-Léger peut revenir à la question qui est la sienne depuis les débuts de son travail sociologique: celle de l'utopie $^{5}$. En monachisme, un article classique de Jean Séguy a balisé le terrain au début des années $1970^{6}$. Mais Séguy écrivait en un temps de «hautes eaux utopiques ", tandis que l'on ne peut aujourd'hui que constater l'étiage, dans les sociétés occidentales en général et jusque derrière les murs des monastères. Le grand projet prophétique d'anticipation du Royaume semble s'être résorbé aux dimensions d'un «art de vivre", à la recherche d'une "petite musique " visant à tenir la note juste dans les rapports avec le divin, avec le cosmos, avec la communauté humaine, avec soi-même. Si D. Hervieu-Léger se refuse néanmoins à signer le permis d'inhumer de l'utopie monastique, c'est qu'à ses yeux l'ouverture eschatologique, toujours présente comme horizon même si le discours est devenu plus modeste, n'autorise pas à confondre l'éthos bénédictin avec les nombreuses formes de micro-sociétés alternatives contemporaines.

Ce faisant, la démarche sociologique croise la réflexion si neuve de G. Agamben, qui a centré un large pan de son travail récent sur la règle et ses implications ${ }^{7}$. Avec une belle audace, comparable sur un autre plan à celle de la sociologue, il a rendu à la liturgie sa place philosophique, montrant qu'il est incohérent de tenir à l'écart le De officiis de la ritualité chrétienne quand on fait l'apologie du De officiis de la sagesse antique. La liturgie, explique-t-il, est au cœur du projet régulier, qui prône l'assimilation intégrale entre vie et norme communautaire, jusqu'à l'émergence de "quelque chose d'inouï et de nouveau, c'est-à-dire une forme-de-vie ${ }^{8}$ ». Cette "loi qui s'indétermine en vie ${ }^{9}$ " ouvre des pistes fécondes, puisque qu'elle est une voie royale pour le dépassement de l'ontologie classique par une pensée du mode d'être. À ce propos, on sait la place qu'Agamben accorde, dans sa lecture de l'Épître aux Romains, à la formule paulinienne «comme si ne pas ", hos me, dans laquelle il voit «le sens ultime de la klesis» (vocation), sous les espèces d'une véritable règle éthique, formulée par exemple en 1 Co $7:$ :User du monde comme

5. D. Hervieu-Léger et B. Hervieu, Des communautés pour les temps difficiles : néo-ruraux ou nouveaux moines, Paris, Centurion, 1983.

6. J. Séguy, «Les sociétés imaginées : monachisme et utopie », Annales ESC, 26, 1971, p. 328-354.

7. G. Agamben, De la très haute pauvreté : règles et forme de vie (Homo sacer, IV, 1), Paris, Payot et Rivages, coll. "Bibliothèque Rivages ", 2011. Notons qu'Agamben donne comme aboutissement à sa recherche le monde franciscain, ce qui est cohérent du point de vue de la régularité, mais fait sortir du cadre monastique à proprement parler.

8. Ibid., p. 8 .

9. Ibid., p. 119. 
en n'en usant pas, car la figure du monde passe ${ }^{10}$ ». Y a-t-il désignation plus claire du propositum monastique en sa substance même? D. Hervieu-Léger se trouve ainsi justifiée d'avoir choisi pour son enquête l'entrée monastique: elle est allée d'emblée vers le lieu géométrique de la nouveauté et de la radicalité chrétienne.

De radicalité il est aussi question dans ce que l'auteur fait apparaître comme le point d'orgue de sa longue recherche: l'enjeu de l'hospitalité. Elle décrit minutieusement les manières très diverses dont les hôtelleries monastiques, en ce début de $\mathrm{XXI}^{\mathrm{e}}$ siècle, s'ouvrent à ceux que les hasards de la vie ont jetés sur les routes et qui n'ont pas tardé à comprendre quel accueil ils pouvaient espérer chez les fils d'un législateur qui enjoignait de reconnaître le Christ lui-même en tout visiteur (Règle, chap. 53). Cette description est comme éclairée par une référence qui, pour venir tout à la fin du livre, n'en est pas moins déterminante, à l'opus magnum du théologien Christoph Theobald, Le Christianisme comme style ${ }^{11}$. Cette référence a un double avantage: permettre de penser l'engagement religieux comme style, dans une configuration où l'on peut considérer le champ social comme structuré par des affrontements entre choix stylistiques antagonistes; identifier, d'autre part, le spécifique chrétien dans un élément que désigne Theobald et qui aurait étonné les ecclésiologues d'antan: l'hospitalité inconditionnelle ${ }^{12}$. Les dépossessions objectives du moine rejoignent ici les dépossessions imposées à tous les "sans » d'une société impitoyable: les sans-statut, les sans-papiers, les sans-abri; la fuga mundi y regagne sa charge politique originelle, à contre-courant des logiques répressives cultivées par la puissance publique. Le choix monastique n'apparaît plus seulement alors comme un engagement archaïsant, fondé sur une nostalgie chrétientaire, mais comme une audace civique, au double horizon de la cité des hommes et de la cité de Dieu, où la Règle dialogue, au moins implicitement, tant avec le modèle abrahamique des trois visiteurs (Gn 18) qu'avec Derrida ou Schérer ${ }^{13}$. Ainsi l'histoire fait-elle, in fine, retour. L'hôtellerie a sans doute toujours été un élément majeur du dispositif monastique, mais sa relation avec le pôle cultuel a constamment évolué, dans une tension permanente entre unicité du geste d'accueil et subtiles diffractions du vœu de pauvreté. Les années récentes ont vu la mise en place d'un nouvel agencement, localement différencié, souvent audacieux, toujours fragile. L'analyse exemplaire qu'en propose D. Hervieu-Léger permet d'entrer dans le laboratoire où s'invente «un témoignage monastique pour une société post-chrétienne». Une telle attention à l'émergent à l'intérieur de cadres institutionnels finement suivis dans la longue durée honore ce que la tradition sociologique a de meilleur.

Alain RAUWEL CéSor (EHESS-CNRS) a.rauwel@wanadoo.fr

10. G. Agamben, Le Temps qui reste: un commentaire de l'Épitre aux Romains, Paris, Payot et Rivages, coll. "Petite bibliothèque Rivages ", 2004, p. 45-49.

11. Éditions du Cerf, 2007 (2 vol.).

12. Sur ce point, A. Rauwel, "L'évangélisme comme injonction à la solidarité », in Wieviorka M. (dir.), Les solidarités, Auxerre, Sciences humaines, 2017, p. 77-88.

13. J. Derrida et A. Dufourmantelle, De l’hospitalité, Paris, Calmann-Lévy, 1997 ; R. Schérer, Zeus hospitalier, Paris, Colin, 1993. 